\title{
The impact of MVDC upon conventional distance protection schemes in hybrid ac-dc distribution networks
}

\author{
L. Hunter *, C. Booth *, A. Dysko *, S. Finney ${ }^{\dagger}$ A. Junyent-Ferré ${ }^{\$}$ \\ * University of Strathclyde, Glasgow, Scotland, \\ \{lewis.hunter.100,campbell.d.booth,a.dysko\}@strath.ac.uk \\ ${ }^{\dagger}$ Electronics and Electrical Engineering, The University of Edinburgh, Scotland, stephen.finney@ed.ac.uk \\ \$Department of Electrical and Electronic Engineering, Imperial College London, UK, adria.junyent-ferre@imperial.ac.uk
}

Keywords: MVDC, Distance Protection, Embedded MVDC, Smart Grid

\begin{abstract}
This paper looks at the protection implications of introducing fully controllable, embedded, medium voltage dc (MVDC) power electronic links into power distribution networks. Studies have indicated, protection notwithstanding, that embedded MVDC can be used to provide economically attractive, enhanced capacity and control of power flows.

Through a series of simulation studies, the impact on distance protection schemes (in terms of reach and response time) resulting from the introduction of a controlled MVDC link have been examined for symmetrical faults. The paper also considers under what conditions a de side fault can be observed from the ac grid.
\end{abstract}

A series of recommendations for system integrators are made.

\section{Introduction}

As they evolve into Distribution System Operators (DSOs), Distribution Network Operators (DNOs) are under pressure to find innovative ways to accommodate rising demand and embedded generation using existing system assets. Several GB (Great Britain) regulator funded innovation projects in the UK have been tasked with determining the advantages and pitfalls of new approaches to increasing flexibility within distribution networks [1] [2].

Embedded medium voltage direct current (MVDC) links could give the future DSO the ability to load network sectors more dynamically to allow significant increases in power flows whilst not exceeding the firm capacity of grid supply point transformers [3] [4]. This idea is being demonstrated by SP Energy Networks through a network innovation competition (NIC) funded project supported by the GB grid regulator, OFGEM (Office for Gas and Electricity Markets) [5]. Large multinational engineering conglomerates are now offering MVDC solutions for both land and sea based applications [6] [7].
The protection required to safeguard controlled links from a dc side fault needs to be fast acting otherwise the power electronic devices are likely to be damaged to the point of replacement. This philosophy is somewhat contrary to traditional ac protection where trip signals may actually be delayed to allow more local circuit breakers to open first.

The approach developed and presented in this paper combines Simulink modelling for the power electronics and the $33 \mathrm{kV}$ network with a distance protection relay modelling tool developed at the University of Strathclyde and verified by National Grid. The studies examine a section of real Scottish distribution network with protection settings derived from the local DNO's long term development statement.

Several fault scenarios have been analysed for different fault locations and operational modes of the controllable MVDC link. For local ac system side faults, converters respond with a fault ride through capability similar to that set out in ENTSO-E for a Type D Power Park [8] (covering connection of medium scale $(\leq 30 \mathrm{MW})$ embedded generation to the transmission system) as grid codes for distribution connected MVDC do not, as yet, exist. For dc side faults, a simplified converter protection strategy is employed.

The primary outcome of this work is to determine whether distance protection remains a valid approach for hybrid ac-dc distribution networks. In particular, the model is used to assess whether a suitably configured distance relay can provide sufficiently fast, backup protection should local ac circuit breakers at a dc link converter station fail.

Studies examine and quantify how the relay reach is affected by downstream MVDC links and how zone boundaries move depending upon the converter's operational mode.

\section{Protection Requirements}

The following subsections will discuss the protection requirements of $33 \mathrm{kV}$ ac distance protection schemes in addition to the fault characteristics of MVDC power electronic converters. 


\subsection{Distance Protection Philosophy}

The tripping time of a distance protection relay is determined by the electrical distance between the relay measurement point and the calculated fault zone. Distance relays rely upon the measurement of both voltage and current from which the fault impedance is derived using ohms law. A fault near the measurement point will result in a lower calculated impedance than a more remote fault. This principle allows operational zones, typically three, to be defined as presented in Figure 1 [9].

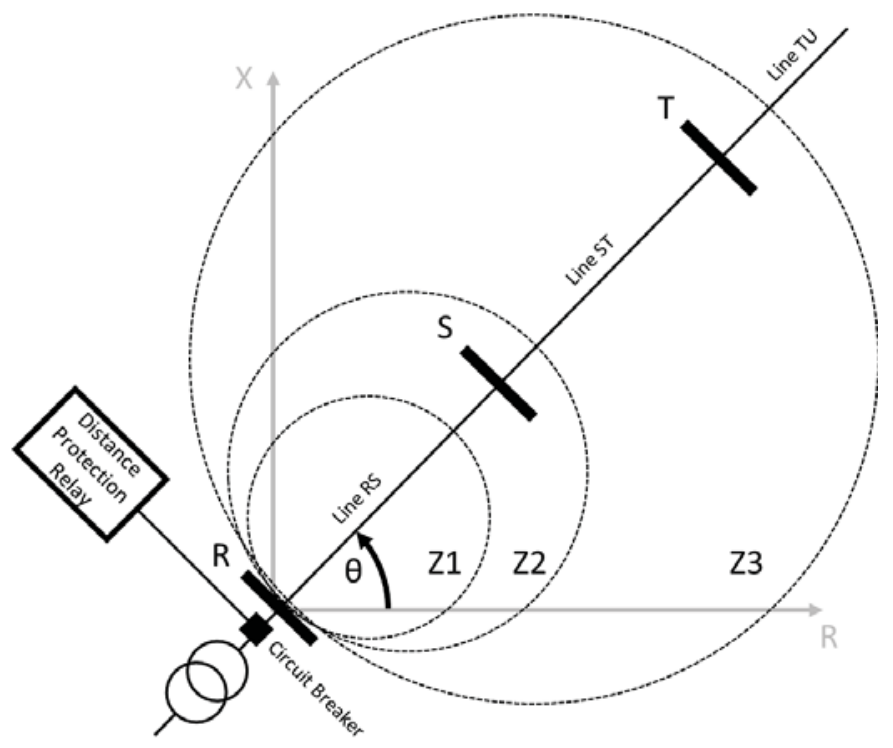

Figure 1: Distance protection zone diagram

- Zone 1 generally reaches to approximately $80 \%$ of the first protected feeder (Line RS) to prevent overreach into Zone 2 which would be caused by errors in the transducers, relay and line parameters' estimation.

- Zone 2 is typically set to $120 \%$ impedance of the protected line (120\% of Line RS).

- Zone 3 typically reaches to $120 \%$ of the combined impedance of lines RS and ST.

Suggested distance protection operation times for a distribution network operator are presented in Table 1. These times are designed to allow discrimination thus allowing a more local circuit breaker to clear the fault first. This time delayed zone approach is designed to reduce the number of customer interruptions associated with a downstream fault.

\begin{tabular}{|c|c|}
\hline Zone & Clearance time $(s)$ \\
\hline $\mathrm{Z} 1$ & 0.150 \\
\hline $\mathrm{Z} 2$ & 0.500 \\
\hline $\mathrm{Z} 3$ & 1.3 \\
\hline
\end{tabular}

Table 1: Maximum clearance times for distance protection schemes as recommended by SSEN [10]

Distance protection can distinguish between eleven possible fault types; $\mathrm{AG}, \mathrm{BG}, \mathrm{CG}, \mathrm{AB}, \mathrm{BC}, \mathrm{CA}, \mathrm{ABG}, \mathrm{BCG}, \mathrm{CAG}$, $\mathrm{ABC}, \mathrm{ABCG}$ where $\mathrm{A}, \mathrm{B}$ and $\mathrm{C}$ are phase conductors and $\mathrm{G}$ is ground. This paper will only consider symmetrical faults due to the early stage nature of this research.

\subsection{Converter Protection Philosophy}

While converters can provide a degree of support to ac side faults their ability to support a dc fault is extremely limited for most topologies. This limitation is primarily due to the freewheel diodes placed across IGBT devices creating a fault path similar to that displayed in Figure 2. The converter effectively becomes an uncontrolled rectifier with current only being limited by the ac side arm inductance [11].

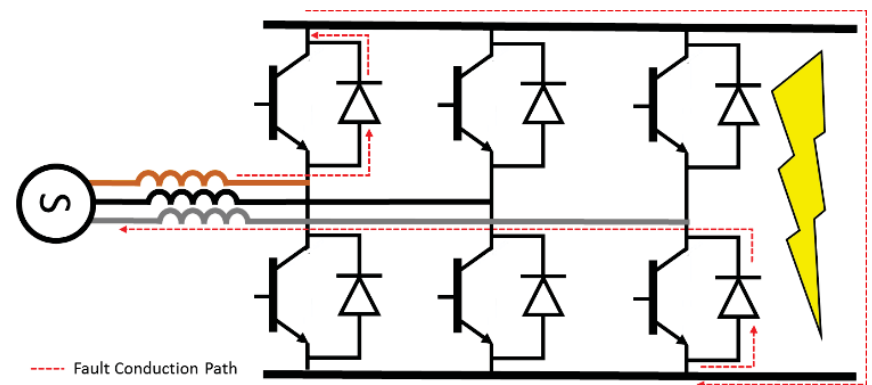

Figure 2: Converter fault path through freewheel diode

The modelled fault response for both ac and dc faults will be outlined.

\section{A. AC side fault strategy}

At present, there are no obligations for a MVDC link to provide any fault ride through capability as there is no requirement in the GB distribution code imposed on DNOs for such a technology of a $<50 \mathrm{MW}$ rating [12].

For the purposes of this study a fault ride through (FRT) capability similar to that set out in ENTSO-E requirements for generators (RfG). A Type D Power Park will be considered (covering connection of medium scale $(\leq 30 \mathrm{MW}$ generation) [13] [8]. The minimum and maximum responses for such a unit are outlined in Figure 3.

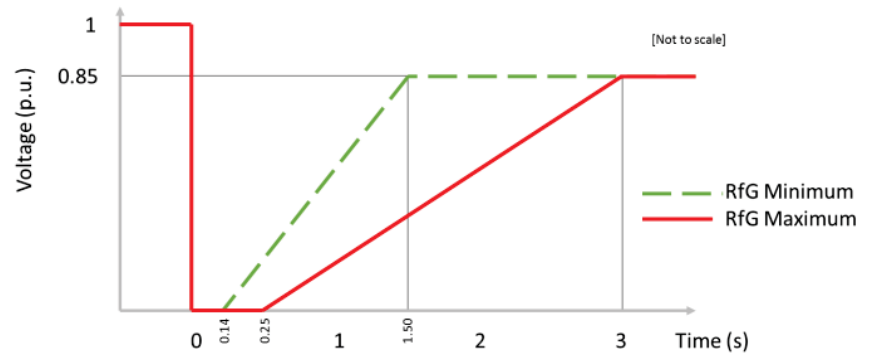

Figure 3: ENTSO-E fault ride through requirements for Type D Power Park [13]

This FRT characteristic of MVDC converters will generally only be capable of delivering fault current into faults on parallel circuits. This is due to the synchronising controller of the MVDC link being unable to lock for series faults as the voltage will be zero at the inverter's point of network coupling. The fault ride through characteristic determines the length of 
time a generating unit should remain connected to the network for a measured terminal voltage.

It is assumed that the converter, where practical, will support network voltage during fault conditions with an infeed of current which is limited to the converter's rating. It is appreciated that other responses are possible.

\section{B. DC side fault strategy (two/three level converters)}

When a dc side fault occurs and is detected by the converter controller the first step taken by the protection system is to block commutation of the switching devices. This prevents the power electronic devices being subjected to high fault currents which would only be limited by converter arm impedances.

The anti-parallel diodes in Figure 2 will conduct once the dc side voltage has collapsed bellow the ac voltage envelope. Bypass devices, often high capacity thyristors, are commonly used to divert current allowing time for protection to operate.

The next action is to open ac side converter circuit breakers to isolate current infeed to the link. This needs to happen quickly to protect the parallel diodes from overheating and critically failing. Upon disconnection at both converter stations, earth switches on the dc sides can be closed via resistor banks to deenergise the link capacitance to a safe voltage.

Should either of these steps fail (converter controller failure, trip signal fail, maloperation of ac side circuit breaker), the converter could be left in a damaging uncontrolled rectifying state [11].

\subsection{Compatibility}

While the time scales involved in protection of ac and dc systems are of differing horizons, studies in this paper aim to;

- For ac faults, investigate whether the distance relay reach (or time) can be adversely affected by an MVDC link

- Determine what the 'seen' impedance of a dc side fault looks like from the ac grid

- Discuss if a suitably configured distance relay can accurately detect and discriminate for a de side fault for backup protection of a MVDC link.

This paper more generally is to look at the performance of ac distance protection in networks with embedded MVDC.

\section{Study Overview}

This section describes the steps carried out to investigate the impact of MVDC upon distance protection devices.

\subsection{Network Modelling}

Network modelling is undertaken using SimPowerSystems components within Simulink. The network section being modelled is based upon small section of $33 \mathrm{kV}$ network found in the south of Scotland where the introduction of embedded
MVDC links has been shown to be promising through simulation [14]. Lines are modelled as lumped element R-L impedances and neglect negative sequence components.

The X/R ratio of the source is 7 for all studies reported in this paper. The system fault infeed level (FL) is 1000 MVA which is in line with GB DNOs' design limit [15].

\subsection{Converter Modelling}

The converter has a notional power rating of $30 \mathrm{MVA}$ operating under a bipolar configuration with voltages of $\pm 27 \mathrm{kV}$. This voltage is based upon the peak ac phase to ground voltage expected under normal operating scenarios in a $33 \mathrm{kV}$ ac network as outlined in equation (1).

$$
V_{d c} \geq \frac{\sqrt{2} \times V_{L L_{R M S}}}{\sqrt{3}}
$$

The converter used for studies is a two level converter which includes protection functionality in line with strategies outlined in section 2.2 [16]. The per-unit impedance of the converter is $50 \%$ (referred to the converter base impedance). Note that this high impedance was used to take the converter transformer [15], filter and arm inductance into account. Power electronic modelling is conducted within the Simulink environment.

\subsection{Dynamic Protection Modelling Controller}

The Dynamic Protection Modelling Controller (DPMC) [17] software package, developed within the University of Strathclyde, is used to determine the impedance of the network seen during steady state and fault conditions. This software was developed to assist National Grid in post fault investigation of power system performance under various transient faults and events.

The software takes time stamped three phase voltages and current values along with a relay setting file. The software calculates which of the distance protection comparators operate and the associated activation time during the system event. DPMC will provide the tripping time of the relay according to the setting file logic. The software also generates an R-X diagram of the event for visual interrogation of the impedance locus.

The GEC Measurements SHNB MicroMho distance protection relay model will be deployed for all simulations in this paper.

\subsection{Methodology}

Figure 4 outlines the steps carried out to model the impact of introducing embedded MVDC links upon distance protection schemes.

Based upon the information gathered from DPMC, recommendations can be made to alleviate potential degradation of distance protection performance. Subsequently, any such performance adjustments can be implemented and 
repeated in DPMC thus allowing such recommendations to be validated.

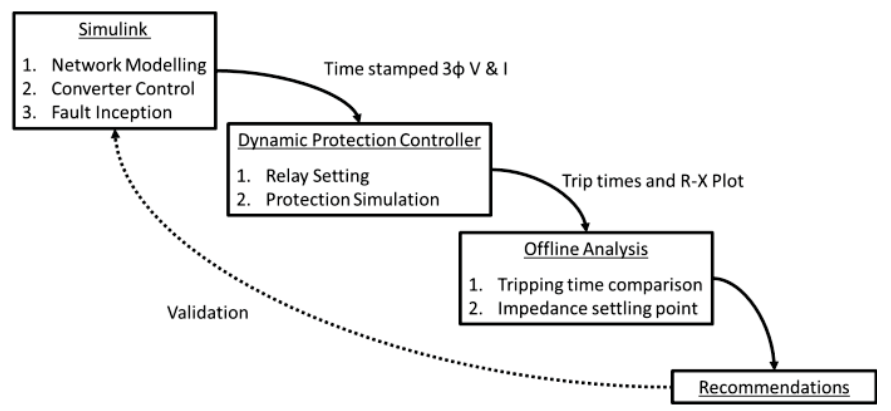

Figure 4: Simulation flow diagram

\subsection{Study Limitations}

It needs to be noted that there are a few limitations to the study. The network model only considers positive sequence lumped R-L element transmission line models rather than a full sequence component model. This is acceptable for this study as three phase symmetrical faults are considered only, but a more detailed line model would be required to study unsymmetrical faults.

Converters are limited to conventional two level voltage source converters (VSC) schemes rather that modular multi-level converter (MMC) style devices. However, it is envisaged that MMC half bridge converters will exhibit a similar ac side response to those modelled due to the diode arrangement within the cells.

\section{Network Studies}

Two main studies are presented in this paper. The first determines whether the reach of a distance protection relay is changed should an embedded dc link with fault ride through capability be introduced. The second study establishes whether the relay can detect a dc side fault. All conductors use the same characteristic impedance of $(0.17+j 0.3) \Omega / \mathrm{km}$. The test network is outlined in Figure 5.

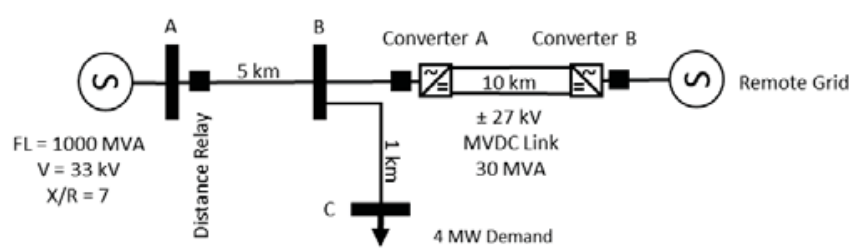

Figure 5: Test network section

\subsection{Relay Setting Parameters}

Relay settings are assigned in accordance with the MicroMho protection manual [18] with zone reach and tripping times specified previously.

A voltage transformer (VT) ratio of $33,000 / 110 \mathrm{~V} / \mathrm{V}$ and a current transformer $(\mathrm{CT})$ ratio of $1750 / 5 \mathrm{~A} / \mathrm{A}$ are selected. The
CT primary current was selected to minimise the effects of transformer saturation on the results.

The angle $(\theta)$ of the mho characteristic is set by considering the ratio between the downstream line reactance and resistance as specified by equation (2).

$$
\theta=\tan ^{-1}\left(\frac{X_{\text {line }}}{R_{\text {line }}}\right)=\tan ^{-1}\left(\frac{0.3}{0.17}\right)=60.5^{\circ}
$$

Key distance zone setting parameters are outlined in Table 2 (note that the impedances are secondary referred therefore taking into account the $\mathrm{CT}$ and VT turns ratios).

\begin{tabular}{|l|l|}
\hline Parameter & Value \\
\hline Mho Phase $\left(^{\circ}\right)$ & 60.5 \\
\hline $\mathrm{Z} 1(\Omega)$ & 1.6 \\
\hline $\mathrm{Z} 2(\Omega)$ & 2.4 \\
\hline $\mathrm{Z} 3(\Omega)$ & 7.2 \\
\hline Z2 Fault Delay (phase \& earth) $(\mathrm{s})$ & 0.5 \\
\hline Z3 Fault Delay (phase \& earth) (s) & 1.3 \\
\hline
\end{tabular}

Table 2: Summary of relay setting parameters

\subsection{Relay Reach Studies - AC Side}

\section{A. No converter fault contribution-ac side fault}

For benchmarking purposes, a three phase to ground fault was thrown at busbar $\mathrm{C}$ after $0.4 \mathrm{~s}$. The load busbar sits between the boundaries of zone 2 and 3. For this study the converter immediately disconnects from the network when a voltage depression is measured. The converter therefore does not contribute any fault current.

The R-X diagram for the fault event is presented in Figure 6 where the fault impedance stabilises in zone 2. Note that axis markers for all R-X diagrams presented are in steps of $1 \Omega$. Table 3 (where $\mathrm{E}$ indicates a fault to earth and $\mathrm{P}$ signifies a phase fault) shows that the relay trips for a zone 2 phase fault at $0.9201 \mathrm{~s}, 0.52 \mathrm{~s}$ after the fault is thrown.

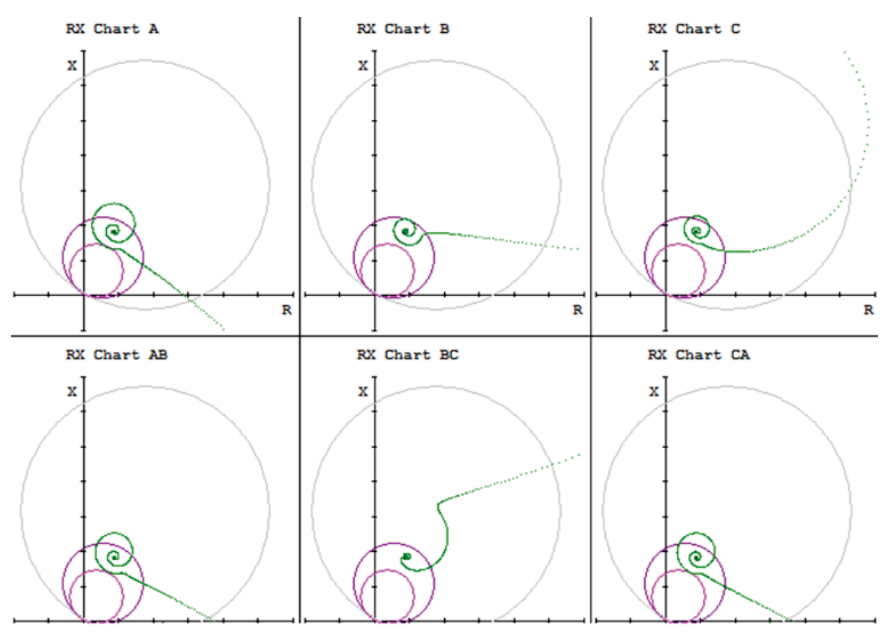

Figure 6: R-X diagram for fault (ABCG) with no infeed 


\begin{tabular}{|c|c|c|c|c|c|c|c|c|}
\hline \multirow{3}{*}{ Scenario } & \multirow{3}{*}{$\begin{array}{c}\text { Time } \\
(s)\end{array}$} & \multicolumn{6}{|c|}{ Element } & \multirow{2}{*}{$\begin{array}{c}\text { Trip } \\
\text { Signal }\end{array}$} \\
\hline & & \multicolumn{2}{|c|}{$\mathrm{Z1}$} & \multicolumn{2}{|c|}{ Z2 } & \multicolumn{2}{|c|}{ Z3 } & \\
\hline & & $E$ & $\mathrm{P}$ & $\mathrm{E}$ & $\mathrm{P}$ & $\mathrm{E}$ & $\mathrm{P}$ & \\
\hline \multirow{3}{*}{$\begin{array}{l}\text { Without } \\
\text { Infeed }\end{array}$} & 0.4179 & & & & $*$ & & & \\
\hline & 0.4201 & & & & $*$ & & $*$ & \\
\hline & 0.9201 & & & & $*$ & & * & * \\
\hline
\end{tabular}

Table 3: Relay log for fault without infeed from MVDC link

\section{B. Converter fault contribution - ac side fault}

From the study conducted it is known that the voltage at the MVDC converter bus bar sits approximately $25 \%$ of nominal during a bolted short circuit. According to the FRT response outlined previously in Figure 3 the converter should remain connected for between 0.5 and 1.1 seconds for such a reduced network voltage. This logic was applied to the converter controller with a random disconnection time between the RFG curves selected.

Table 4 shows that the relay trips for a zone 3 fault in this instance at $1.72 \mathrm{~s}, 1.32$ seconds after fault inception. Figure 7 displays the R-X component of the event where the fault settles just outside zone 2 .

\begin{tabular}{|c|c|c|c|c|c|c|c|c|}
\hline \multirow{3}{*}{ Scenario } & \multirow{6}{*}{ Time } & \multicolumn{5}{|c|}{ Element } & \multirow{2}{*}{ Trip } \\
\cline { 3 - 8 } & & \multicolumn{2}{|c|}{ Z1 } & \multicolumn{2}{|c|}{$\mathbf{Z 2}$} & \multicolumn{2}{|c|}{$\mathbf{Z 3}$} & Signal \\
\cline { 3 - 8 } & & $\mathrm{E}$ & $\mathrm{P}$ & $\mathrm{E}$ & $\mathrm{P}$ & $\mathrm{E}$ & $\mathrm{P}$ & \\
\hline With & 0.4200 & & & & & & $*$ & \\
\cline { 2 - 8 } Infeed & 1.7222 & & & & & & $*$ & $*$ \\
\hline
\end{tabular}

Table 4: Relay log for fault with infeed from MVDC link

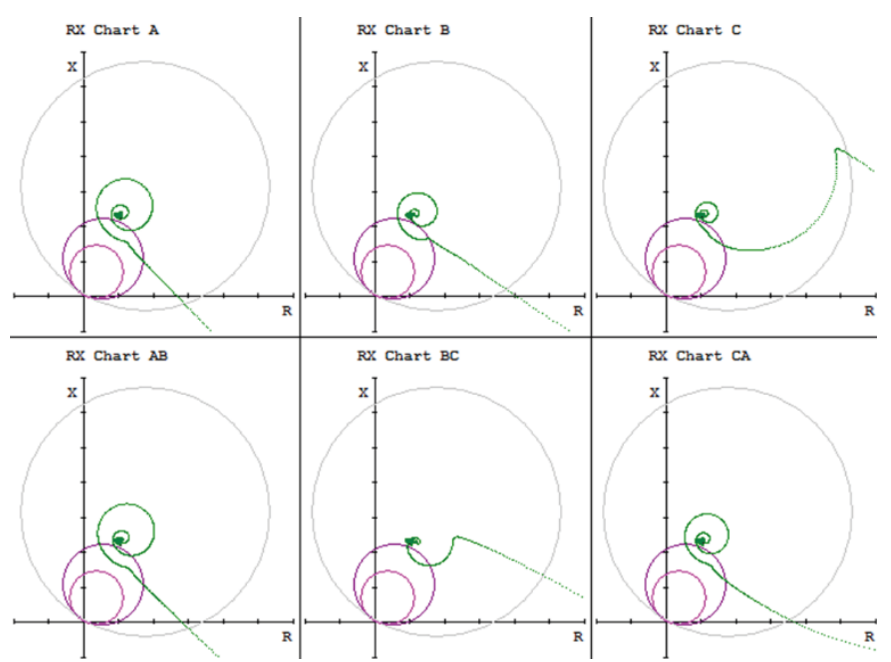

Figure 7: R-X diagram for fault (ABCG) with MVDC infeed

It is observed that the fault ride through characteristic of the MVDC converter used in this study has the ability to support the voltage near a branch ac side fault resulting in the calculated fault zone shifting from zone 2 to zone 3; thus delaying disconnection time by $800 \mathrm{~ms}$.

\subsection{Side Pole to Pole Fault Studies}

For this fault type it is assumed that the local MVDC converter fails to break during dc side faults, and the remote converter disconnects from the ac network within two cycles $(40 \mathrm{~ms})$ of fault inception.

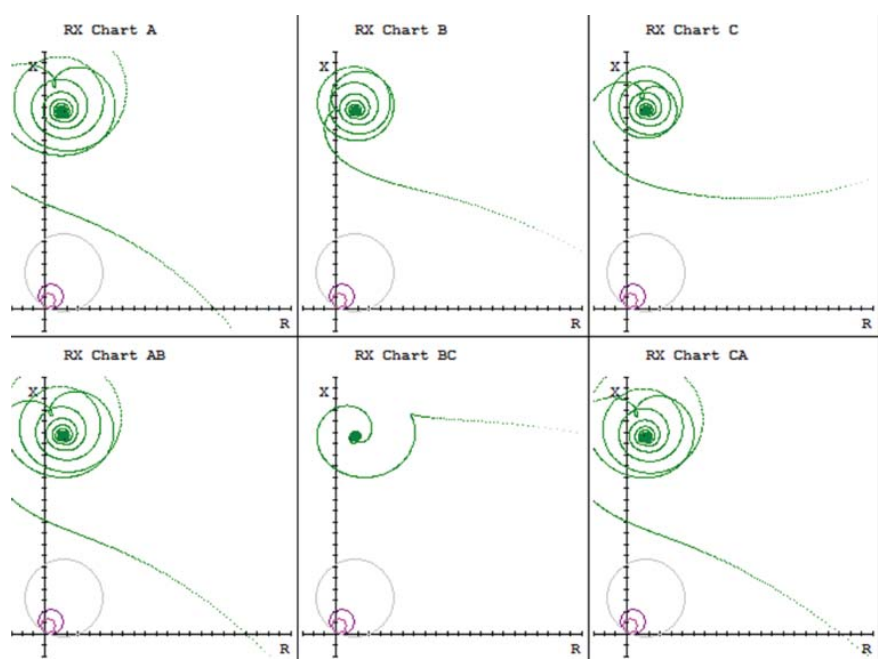

Figure 8: R-X diagram for a pole to pole fault on dc link

The fault trace lies significantly outside of all the protection zones of the distance protection relay. However, the fault does reach a steady state at $(1.5+j 17.5) \Omega$.

The steep angle associated with the impedance locus is due to the significant inductance placed between the ac and dc systems used to protect the MVDC converter during dc side faults but also to act as a harmonic filter.

It is hypothesised that a zone 4 setting of a modern digital relay configured with quadrilateral characteristic may be able to provide sufficiently quick backup protection to an embedded MVDC link for a de side fault.

\section{Discussion and Future Works}

From the studies conducted in this paper it is proposed that a form of accelerated distance protection may offer an innovative method of backing up main converter protection for dc side faults. While further work is required to validate this approach fully across multiple operating scenarios, initial findings appear promising.

When considering ac side faults it is observed that the converter can cause the relay to incorrectly identify fault location due to the raised feeder voltage (distance relay underreach). This has the potential to increase fault clearance times which is in line with other published studies looking at the effects of distributed generation (DG) upon distance protection schemes [19].

It is worth noting that faults which are in series with the MVDC converters are unlikely to affect clearance times as the voltage collapse associated with the fault results in the phase locked loop controller of the converter losing synchronism, thus disconnecting the link from the network. 
A wider range of operating conditions must also be considered to ensure the sensitivity (the ability to identify faults on the system which appear close to normal operating conditions) of the proposed protection approach. Hardware in the loop simulations are currently undergoing investigation using an RTDS (Real Time Digital Simulator) system housed within the University of Strathclyde.

While these studies have been conducted upon a distribution network the findings could also potentially be translated to HVDC transmission scale protection.

For MVDC to be a viable solution to unleashing capacity on the medium voltage networks the modularity of the converters needs to increase and standardise to reduce costs. Remote backup protection of an MVDC link is likely to give grid operators more confidence when deploying such a solution upon their networks.

\section{Conclusion}

This paper has presented a methodology to investigate potential impacts from MVDC links on power system protection performance.

The need for increased control in distribution networks is widely accepted. The role and effectiveness of existing network protection strategies must be validated for topologies where power electronic links are introduced. Modelling via software has identified that the reach of a distance relay could be adversely affected by embedded links.

Through studies presented in this paper it has also been shown that conventional distance protection has the ability to observe faults upon a dc link should the converter topology not have dc fault blocking capability.

The use of existing distance protection relays to act as a backup protection for embedded MVDC links may be attractive for the future DSO. Further studies are currently being carried out both in real-time and offline simulation environments to verify further the appropriateness of using conventional ac distance relays as backup protection for an embedded MVDC link.

\section{Acknowledgements}

This work has been supported through the EPSRC Centre for Doctoral Training in Future Power Networks and Smart Grids (EP/L015471/1).

\section{References}

[1] Scottish and Southern Electricity Networks, "Northern Isles New Energy Solutions (NINES)," [Online]. Available: https://www.ssepd.co.uk/NINES/. [Accessed June 2018].

[2] SP Energy Networks, "Accelerating Renewable Connections (ARC)," [Online]. Available: https://www.spenergynetworks.co.uk/pages/arc_accelerati ng_renewable_connections.aspx. [Accessed June 2018].

[3] F. Mura and R. W. De Doncker, "Design Aspects of a Medium-Voltage Direct Current (MVDC) Grid for a University Campus," in 8th International Conference on Power Electronics - ECCE Asia, Korea, 2011.

[4] TNEI, "MVDC Technology Study - Market Opportunities and Economic Impact," 2015.

[5] SP Energy Networks, "Angle DC," [Online]. Available: https://www.spenergynetworks.co.uk/pages/angle_dc.aspx . [Accessed December 2016].

[6] Siemens, "MVDC Plus," 2017. [Online]. Available: siemens.com/mvdc.

[7] GE, "GE Supports Power Grids of the Future with Europe's First MVDC Link," GE Power Conversion, 16 May 2017. [Online]. Available: https://www.gepowerconversion.com/press-releases/gesupports-power-grids-future-europe $\% \mathrm{E} 2 \% 80 \% 99$ s-firstmvdc-link. [Accessed July 2018].

[8] "Network code on requirements for grid connection of generators (L112)," Official Journal of the European Union, 2016.

[9] GE Power Management, "Distance Relays Fundamentals," GE MULTILIN, Ontario, 2002.

[10] SSEN, "Long Term Development Statement," 2016.

[11] F. P. Page, Analysis in Circuit Breaker Performance Requirements for High-Voltage DC Networks, 2016.

[12] DCODE, "The Distribution Code for licensed DNOs of Great Britain - Issue 29," February 2018.

[13] National Grid Electricity Transmission plc, "Fault Ride Through: ENTSO-E Requirements for Generators," National Grid Electricity Transmission plc, 2013.

[14] L. Hunter, C. Booth, A. J. Ferre and S. Finney, "MVDC for enhanced utility scale distribution power delivery and control," in 52nd International Universities Power Engineering Conference (UPEC), Heraklion, 2017.

[15] SP Energy Networks, "Long Term Development Statement," 2016.

[16] A. Florida-James, S. Yana, A. Emhemed and G. Burt, "Investigation of a decentralised control strategy for grid frequency support from DC microgrids.," in The 7th International Conference on Renewable Power Generation (RPG 2018), Copenhagen, Denmark, 2018.

[17] A. Dyśko, J. R. McDonald, G. M. Burt, J. Goody and B. Gwyn, "Integrated Modelling Environment : A Platform for Dynamic Protection Modelling," IEEE, New Orleans, LA, USA, 1999.

[18] GEC Measurements, "MicroMho - Static Distance Protection Relay," The General Electric Company p.l.c. England, Stafford, 1984.

[19] A. K. Pradhan and G. Joos, "Adaptive Distance Relay Setting for Lines Connecting Wind Farms," IEEE Transactions on Energy Conversion, vol. 22, no. 1, pp. $206-213,2007$. 